\title{
Les formations ouvertes à distance, leur dynamique et leur contribution en contexte africain
}

\author{
Thierry Karsenti ${ }^{1}$ - Simon Collin ${ }^{2}$ \\ 1. Université de Montréal \\ Faculté des sciences de l'éducation \\ C.P. 6128, Succursale Centre-ville \\ Montréal (Québec) H3C 3J7 \\ thierry.karsenti@umontreal.ca \\ 2. Université du Québec à Montréal \\ Département de didactique des langues \\ Faculté des sciences de l'éducation, \\ C.P. 8888, Succursale Centre-ville \\ Montréal (Québec) H3C 3P8 \\ collin.simon@uqam.ca
}

\begin{abstract}
RÉSUMÉ. Le potentiel éducatif des formations ouvertes à distance (FOAD) revêt un intérêt tout particulier en Afrique, où l'enseignement supérieur est confronté à de multiples contraintes (Gioan, 2007). Le développement des FOAD est toutefois confronté à des difficultés au niveau de l'équipement informatique, de l'organisation et des compétences. Dans cette perspective, cet article expose les résultats partiels d'une étude mixte de trois ans (2007-2010) portant sur les formations ouvertes à distance (FOAD) offertes par l'Agence universitaire de la francophonie (AUF). Il aborde, au moyen d'une analyse qualitative d'entrevues, la contribution des FOAD pour le développement des professionnels africains en ayant pour objectif de mieux comprendre les dynamiques à l'œuvre lors du suivi d'une FOAD par des étudiants africains.

ABSTRACT. In Africa, where higher education systems are coping with multiple constraints (Gioan, 2007), open and distance learning (ODL) has enormous potential. However, a number of obstacles must be overcome, including insufficient computer equipment, organization, and skills. Against this background, this article presents the partial results of a three-year (20072010) mixed methods study of ODL programs offered by the Agence universitaire de la Francophonie $(A U F)$. Based on a qualitative analysis of interviews, it examines how ODL can contribute to the professional development of Africans. The aim is to gain a deeper understanding of the dynamics at play when African students follow an ODL program.
\end{abstract}

MOTS-CLEFS : FOAD, Afrique, perceptions, étudiants.

KEYWORDS: ODL, Africa, perceptions, students.

DOI:10.3166/DS.9.493-514 @ Cned/Lavoisier 2011

D\&S - 9/2011. Où va la distance ?, pages 493 à 514 


\section{Introduction}

Les formations ouvertes à distance (FOAD) contribuent fortement au développement actuel de l'enseignement supérieur (Harry, 1999), bien que leur progression soit en dessous des calculs prévisionnels (OCDE, 2006). Elles peuvent être définies comme « une démarche qui vise à élargir l'accès aux services éducatifs et de formation en permettant aux apprenants de franchir les obstacles que représentent l'espace et le temps et en proposant des modalités d'enseignement souples aux individus comme aux groupes d'apprenants » (UNESCO, 2006). Leur potentiel éducatif revêt un intérêt tout particulier en Afrique, où les universités sont confrontées à la «triple contrainte d'une forte croissance des effectifs de l'enseignement supérieur, de marges budgétaires réduites et d'un marché de l'emploi peu porteur» (Gioan, 2007, p. vii). Il en résulte un retard considérable de l'enseignement supérieur africain: alors que le taux brut de scolarisation dans l'enseignement supérieur se situe à $26 \%$ au niveau mondial en 2007, celui de l'Afrique est de $6 \%$ (Institut statistique de l'UNESCO, 2009, p. 14). À cet égard, les FOAD sont souvent perçues comme une alternative intéressante. Leurs modalités pédagogiques permettent d'accueillir un plus grand nombre d'étudiants pour un coût moins élevé que le présentiel (Brossard et Foko, 2007). Leur flexibilité spatiotemporelle contribue également à diversifier la clientèle attendue, en intégrant notamment des professionnels déjà en fonction en plus des étudiants en formation initiale (Gilbert, 2000). À ce titre, les FOAD sont susceptibles de contribuer de façon efficace au développement d'une main-d'œuvre africaine qualifiée, ce qui explique pourquoi elles sont par exemple mentionnées dans le Plan d'action de la Seconde décennie de l'éducation pour l'Afrique (2006-2015) en lien avec l'enseignement supérieur. Le développement des FOAD est toutefois confronté à des difficultés au niveau de l'équipement informatique, de l'organisation et des compétences professionnelles. Dans cette perspective, cet article expose les résultats partiels d'une étude mixte de trois ans (2007-2010) portant sur les formations ouvertes à distance (FOAD) offertes par l'Agence universitaire de la Francophonie (AUF). Il aborde, au moyen d'une analyse qualitative d'entrevues, la contribution des FOAD pour le développement des professionnels africains en ayant pour objectif de mieux comprendre les dynamiques à l'œuvre dans le suivi des FOAD.

\section{Contexte}

De par le nombre de politiques, d'initiatives locales, nationales et internationales et d'études empiriques disponibles sur le sujet, il est possible d'avancer que les TIC constituent assurément un objet d'intérêt en contexte africain. Leur rôle est pourtant perçu de manière variable. Ainsi, certaines études scientifiques sur le sujet semblent développer un argumentaire davantage idéologique qu'empirique. En filigrane d'un tel argumentaire se trouve l'idée que les TIC formeraient un vecteur inconditionnel et automatique de développement socio-économique des pays africains, ce qui semble 
relever d'une vision idéalisée des TIC, d'un «mythe de la technique » (Tiemtoré, 2007, p. 9). D'autres études adoptent le point de vue inverse et avancent que les TIC ne sont qu'un aspect de plus contribuant à renforcer les inégalités et les rapports de force en présence (voir par exemple Albirini, 2008). Entre ces deux extrêmes, relevant de logiques technophobes et technophiles (Sfez, 2002), certaines études optent pour une approche plus rationnelle et s'appliquent à considérer les TIC pour ce qu'elles sont, à savoir, des outils au service d'une population donnée, et qui la modifient en retour (Polikanov et Abramova, 2003). C'est dans cette optique que nous abordons les TIC dans cette étude, en reconnaissant le potentiel des FOAD pour le développement des pays africains, mais également les limites auxquelles elles se heurtent, ce qui fait l'objet des sections suivantes.

\section{Potentiel des FOAD pour le développement des professionnels africains}

En contexte africain, les FOAD semblent susceptibles de contribuer à freiner une tendance supposément négative pour le développement des professionnels qualifiés, à savoir, leur migration vers les pays du « Nord », ce qui est communément appelé la fuite des cerveaux. Cette dernière a longtemps été décriée comme un contredéveloppement pour les pays africains, dû au fait qu'elle diminuerait leur capital humain au profit de celui des pays occidentaux (Docquier, 2007). Cette position est de plus en plus nuancée par certains auteurs (voir par exemple, Meyer et Charum, 1995 ; Cervantes et Guellec, 2002), lesquels avancent que la migration des professionnels africains a aussi des impacts positifs pour les sociétés d'origine, notamment au moyen des TIC. Docquier (2007) cite, entre autres, «les transferts de fonds, le retour des migrants après avoir acquis des connaissances à l'étranger, la formation de réseaux de contacts commerciaux, les effets des perspectives de migration sur la formation de capital humain, les effets de gouvernance et de discrimination ethnique » (p. 73). Dans cette perspective, la fuite des cerveaux ne correspondrait pas uniquement à une «expropriation intellectuelle», comme elle a été traditionnellement dépeinte, mais à un phénomène complexe de pertes et de gains en interaction les uns avec les autres et dans le temps, ce qui inviterait à reconsidérer les cadres de référence utilisés jusque-là.

C'est dans ce contexte que nous situons le potentiel des FOAD pour les pays africains, en tant que dispositifs de formation flexibles permettant aux apprenants de suivre une formation professionnelle externe à leur milieu socioculturel immédiat tout en y restant, ce qui contribuerait à faciliter le réinvestissement de leurs compétences professionnelles dans leur contexte local. Dès le commencement des FOAD, certains auteurs ont perçu leur potentiel pour le développement africain. Ainsi, Jacquinot souligne déjà en 1993 que les FOAD sont susceptibles « d'éviter la fuite des cerveaux et les difficultés inhérentes à la transplantation culturelle» (p. 57), argument encore en vigueur aujourd'hui (voir, par exemple, Moughli et al., 2008). Les FOAD recèlent donc un potentiel non négligeable pour le développement des professionnels africains. Elles se heurtent toutefois à certains défis. 


\section{Défis liés aux FOAD en Afrique : une question de distance?}

Pour présenter les défis liés aux FOAD en contexte africain, il nous semble opportun d'exploiter le cadre proposé par Jacquinot (1993), pour qui le degré d'accès et d'usage des TIC est attribuable à une série de distances, que nous revisitons pour l'adapter au cas d'apprenants africains suivant des FOAD majoritairement dispensées par des universités « du Nord» :

- la distance spatiale : parce qu'il s'agit du sens littéral du terme « distance », ce type de distance est sans doute celui qui a le plus motivé la création et l'essor des FOAD. En effet, suivre une FOAD vise en premier lieu à se défaire d'une contrainte spatiale : celle d'être physiquement présent aux côtés de l'enseignant. En contexte africain, la distance spatiale permise par les FOAD permet de compenser l'offre réduite de formation en présentiel. Elle peut toutefois devenir une barrière si les apprenants africains ne sont pas nécessairement à proximité d'un équipement technologique nécessaire au suivi d'une FOAD. Y participer implique alors des déplacements physiques dans des lieux technologiquement équipés, tels que les Centres numériques d'apprentissage de l'AUF ou les cybercafés (Karsenti et Collin, 2010). En ce sens, le dépassement de la distance spatiale initialement permis par les FOAD entraîne en contrepartie une nouvelle distance spatiale pour ceux dont l'équipement technologique n'est pas à proximité ;

- la distance temporelle : comme le rappelle Jacquinot déjà en 1993, les FOAD se caractérisent également par une double distance temporelle : la gestion du temps de formation est généralement plus flexible qu'en présentiel, ce qui demande en contrepartie aux apprenants une plus grande responsabilité et des capacités d'autorégulation quant à leur rythme de formation; par ailleurs, la communication partiellement ou entièrement asynchrone des FOAD entre les apprenants et les enseignants peut également constituer une contrainte supplémentaire pour certains apprenants, que l'interaction en face à face en salle de classe ne poserait pas ;

- la distance technique : ce type de distance renvoie à la maîtrise des technologies nécessaires au suivi d'une FOAD. En effet, la FOAD introduit une instrumentation technologique supplémentaire à ce que propose le présentiel et demande par conséquent la maîtrise de cette instrumentation. En Afrique, la présence timide des TIC tend à limiter le développement des compétences technologiques des apprenants suivant une FOAD. À cette première distance technique peut s'en ajouter une deuxième : celle du dysfonctionnement ou de la vétusté du matériel technologique, lorsqu'il est accessible (voir la distance spatiale sur ce dernier point) ;

- la distance socioéconomique: ce type de distance renvoie au niveau socioéconomique des apprenants, lequel détermine en grande partie les ressources matérielles (équipement technologique, financement) disponibles pour suivre une FOAD. Dans le contexte africain, plusieurs auteurs (Oladele, 2001 ; Intsiful, Okyere et Osae, 2003; Selinger, 2001; Tunca, 2002) relèvent le manque d'outils, la logistique inopérante, l'insuffisance ou le défaut d'infrastructures technologiques. La distance socioéconomique avec les FOAD dispensées par les pays « du Nord» est 
donc non négligeable et constitue à ce titre une des barrières principales des FOAD en Afrique ;

- la distance socioculturelle : ce type de distance est loin d'être anodin. Plusieurs auteurs ont déjà pointé du doigt l'ethnocentrisme des sciences de l'éducation, lesquelles sont essentiellement fondées dans la perspective d'un individu blanc occidental, ce qui exclut d'emblée ceux qui ne répondent pas à ce profil et aux valeurs qui le sous-tendent (Akkari, 2002; Mott, 2006). À titre d'exemple, la capacité des individus (élèves ou professionnels) à travailler individuellement ou collectivement est en grande partie déterminée par leur culture d'origine, comme l'ont montré plusieurs études (ex. : Allen et Boykin, 1992 ; Cassady et al., 2004). Ce point est également vrai pour l'ingénierie des FOAD (Blanchard et Frasson, 2005 ; Henderson, 1996) et devient particulièrement important lorsque les FOAD ont une portée internationale ;

- la distance pédagogique : «La » dernière distance est d'ordre pédagogique. Parce que les pratiques pédagogiques sont fortement déterminées par la culture dans laquelle elles prennent place, la distance pédagogique peut être vue comme un sousensemble de la distance socioculturelle. Ainsi, la pédagogie basée sur l'enseignement transmissif et magistral est encore très présente dans les systèmes éducatifs africains, ce qui peut s'avérer difficilement compatible avec le paradigme constructiviste qui prévaut dans la conception des FOAD occidentales.

Ce bref aperçu nous permet d'avancer que les FOAD ont un potentiel intéressant pour participer au développement des professionnels africains sans que l'on sache actuellement si les défis rencontrés, que nous avons abordés en termes de distances, permettent d'actualiser ce potentiel ou non. Pour tenter d'y répondre, cet article cherche à mieux comprendre les dynamiques à l'œuvre dans le suivi des FOAD en contexte africain.

\section{Méthodologie}

Dans le cadre de cette étude, nous avons ciblé les FOAD soutenues par l'Agence universitaire de la francophonie (AUF). Au total, il s'agit d'une cinquantaine de FOAD de niveau licence ou maîtrise offertes par des institutions d'enseignement supérieur de Belgique, du Burkina Faso, du Cameroun, du Québec, de France, du Sénégal et de Tunisie. Le rôle de l'AUF est d'encourager des étudiants et des professionnels africains en formation continue à suivre une FOAD, tout en restant dans leur pays et en continuant à travailler. Notre étude a donc ciblé les apprenants africains inscrits à une des FOAD soutenues par l'AUF.

Plus précisément, nous avons fait passer des entrevues téléphoniques individuelles semi-dirigées (avec la téléphonie IP) à 24 participants ayant manifesté leur intérêt lors du remplissage d'un questionnaire diffusé préalablement. En sélectionnant les participants, nous nous sommes assurés d'une certaine répartition de notre échantillon suivant différents paramètres, tels que l'âge, le sexe, le pays de résidence et le degré 
d'avancement dans la FOAD suivie (i.e. participants suivant, au moment des entrevues, une FOAD ; participants ayant terminé, au moment de l'entrevue, une FOAD). Au final, notre échantillon se compose de 10 femmes et de 14 hommes, âgés de 23 à 47 ans résidant dans 15 pays différents. Douze d'entre eux suivaient une FOAD au moment de l'entrevue alors que les 12 autres avaient déjà terminé leur formation. Précisons que notre étude ne visait pas à différencier les participants suivant leur origine ethnique au sein de l'Afrique, malgré la richesse culturelle qui prévaut dans ce continent. Par conséquent, aucune analyse comparative n'a été effectuée sur le plan ethnique. Par ailleurs, comme les FOAD concernées étaient nombreuses et que l'accent a été mis sur les apprenants, nous n'avons pas souhaité détailler avec précision la conception propre à chaque FOAD observée. Cette étude se veut donc globale et consiste à dresser un portrait d'un profil d'apprenants (les apprenants africains) vis-à-vis d'un groupe de FOAD (celles soutenues par l'AUF).

Avant de procéder aux entrevues, nous avons élaboré deux protocoles : un à l'intention des participants suivant, au moment de l'entrevue, une FOAD soutenue par l'AUF ; l'autre à l'intention des participants ayant terminé une FOAD soutenue par l'AUF. Les protocoles ont été élaborés en fonction des thématiques suivantes : profil sociologique et technologique; motivation à suivre une FOAD ; représentations de l'enseignement à distance ; difficultés et satisfaction à l'égard de la FOAD; bénéfices tirés des diplômes acquis, ce dernier point ne s'adressant qu'aux 12 participants ayant terminé leur FOAD au moment de l'entrevue. Chaque protocole a été prétesté puis bonifié auprès d'un participant suivant ou ayant terminé, au moment de l'entrevue, le suivi d'une FOAD soutenue par l'AUF.

Les passations d'entrevues, d'une durée moyenne de 45 minutes, ont eu lieu à l'été 2009 via le logiciel de téléphonie IP Skype. Nous avons par la suite procédé à une analyse qualitative de contenu (L'Écuyer, 1990 ; Huberman et Miles, 1991) avec le logiciel QDA Miner. Elle a fait intervenir les phases suivantes :

- lecture du verbatim ;

- élaboration d'une grille préliminaire de codes à partir des différents thèmes émergeant de la lecture du verbatim ;

- codage de $25 \%$ du verbatim et bonification itérative de la grille de codes en conséquence ;

- contre-codage des $25 \%$ du matériel codé et bonification de la grille de codes en conséquence ;

- application du codage au reste du matériel ;

- classement des codes en catégories ;

- interprétation. 


\section{Résultats}

Les résultats sont présentés par ordre chronologique : seront d'abord évoqués les aspects intervenant au commencement des FOAD, puis les aspects pédagogiques, technologiques, administratifs et socioprofessionnels et enfin les retombées des FOAD pour les participants.

\section{Commencement des FOAD}

Le commencement des FOAD peut être abordé suivant la motivation initiale des apprenants, leurs préconceptions et le processus de familiarisation qu'ils semblent vivre au niveau pédagogique et technique.

\section{Motivations initiales à suivre une FOAD}

Le développement de compétences professionnelles est de loin la première motivation évoquée par les participants. Il s'applique généralement à des professionnels déjà en poste qui souhaitent poursuivre leur développement :

$\mathrm{P} 2^{1}$ : « Je ne cherche pas à valider un diplôme, mais tout simplement à acquérir une certaine compétence, certains atouts, à rénover mon cursus de formation professionnelle. »

P8 : «L'objectif premier [...] c'est d'approfondir les connaissances que j'avais et puis de découvrir de nouveaux horizons. »

Dans cette perspective, la motivation à suivre une FOAD peut s'inscrire dans un projet de développement professionnel plus large :

F3 : «Mes ambitions [...] pour plus tard, c'est justement de pouvoir réaliser des ressources multimédias et des cédéroms interactifs en arabe pour les petits écoliers. »

F12 : «Je voulais voir comment, dans le cadre d'un projet d'innovation, je peux proposer des solutions qui peuvent être source de création de valeurs. »

Pour autant, l'inscription à une FOAD n'est pas purement désintéressée. Pour la plupart des participants, elle vise à répondre à des lacunes de formation ou à des besoins conjoncturels :

P1: «Comme je suis enseignante, j'étais sensibilisée à l'importance de la technique de communication parce que dans notre culture universitaire malheureusement, on ne reçoit que la formation purement agronomique et le côté pédagogique est totalement négligé. »

1. Tout au long du texte, la lettre $P$ réfère aux participants poursuivant, au moment de l'entrevue, une FOAD soutenue par l'AUF alors que la lettre F renvoie aux participants ayant fini, au moment de l'entrevue, une FOAD soutenue par l'AUF. 
P5 : «Actuellement, avec l'évolution des nouvelles technologies et surtout avec le niveau actuel des étudiants, nous n'avons pas assez de formation à notre disposition alors j'ai sauté sur la première occasion qui m'a été présentée. »

À ce titre, la motivation à suivre une FOAD ne représente pas uniquement une «valeur ajoutée ». Elle vient aussi répondre à un manque à gagner du contexte professionnel.

Parallèlement au développement professionnel, qu'il soit désintéressé ou rendu nécessaire, l'augmentation de l'employabilité constitue un autre motif d'inscription aux FOAD :

F7 : «Je voulais au départ être qualifié, avoir des diplômes non seulement sur le plan national, mais aussi sur le plan international. Ainsi, ils pourraient me permettre d'avoir une plus grande chance sur le marché de l'emploi.

P3 : «Je regarde un objectif final que je me suis fixé, c'est-à-dire de gagner un diplôme. »

\section{Préconceptions des FOAD par les apprenants}

Concernant les préconceptions des participants vis-à-vis des FOAD, deux cas de figure ont été identifiés :

- soit les participants n'ont pas de préconceptions. Autrement dit, ils n'ont pas une idée claire de ce qu'est une FOAD avant d'en faire l'expérience :

P5 : «Avant, je ne savais pas comment ça allait se passer. »

F4 : «Vraiment, j'étais un peu perdu, mais je savais que j'allais découvrir des choses que je n'avais jamais vues. $\gg$

F6 : «Au début, je ne donnais aucun crédit aux formations à distance puisque c'était quelque chose que je ne connaissais pas. »

- soit les participants entretiennent de préconceptions erronées :

P1 : «C'était pour moi de la formation à distance, donc on est très libre, on n'est pas contrôlé, on peut faire le cours quand on veut. »

P7 : «C'est pas comme je l'avais pensé, où c'était assez flexible et assez de laisser-aller, où je pouvais suivre à mon rythme. »

Nous pouvons alors poser l'hypothèse que le début d'une FOAD exige des apprenants un ajustement de leur conception initiale, en plus d'une familiarisation au déroulement des FOAD, comme nous pouvons maintenant le voir.

Un processus de familiarisation

Le suivi d'une FOAD semble correspondre à un processus qui demande une double familiarisation. La première est de nature technologique : 
P4 : «Au début, je n'avais pas assez de notions sur les outils avec lesquels on travaille. [...] Après un moment, tout s'est bien passé. »

P1 : «Malheureusement, avec la formation que j'ai vécue, on est passé presque directement sans avoir suffisamment le temps de se familiariser avec les outils de l'informatique ou bien la plateforme. $\gg$

F4 : «On n'a pas eu suffisamment de formation là-dessus. »

P12 : «Ce n'est pas évident parce que [...] il y a des trucs techniques, des choses à télécharger pour pouvoir entrer en contact.

P4 : «Pour mon cas, c'est dur parce que l'informatique vraiment pour mon cas, c'est dur, mais je m'y habitue. »

Ce premier type de familiarisation semble toutefois varier d'un participant à l'autre suivant leurs compétences informatiques et celles de leurs proches :

P10 : «Ça [la prise en main de la plateforme] n'a pas été long, ça n’a pas été long parce que $\mathrm{j}$ ' ai suivi une formation en informatique. »

P4 : «J'ai ma petite sœur qui est ingénieure qui m’aide aussi. »

Le deuxième type de familiarisation intervenant dans le suivi d'une FOAD est d'ordre pédagogique. Au-delà de la familiarisation technique, suivre une FOAD implique de nouvelles modalités pédagogiques auxquelles les apprenants ne sont pas toujours accoutumés :

F1 : «Certains, quand il y a des machines, des fois, ils sont gênés de parler à une machine ou d'écrire dans une machine. [...] Donc il y a une nouvelle culture qui s'introduit. Il y en a qui ne sont pas habitués à ça. »

P1 : «Avec le travail en collaboration, généralement, on n'est pas habitué à travailler en équipe. »

Cette double familiarisation renvoie à l'importance d'introduire les apprenants au fonctionnement, tant technologique que pédagogique, des FOAD avant le début des cours :

F2 : «Le Centre AUF organise une rentrée universitaire où tout le monde est convoqué. Vous avez une idée précise de toutes les personnes qui vont participer à la formation et, à partir de là, ça te permet maintenant d'avoir des connaissances que tu n'avais pas connues avant et des personnes sur lesquelles tu peux aussi te reposer. »

P4 : «C'est vrai que nos encadrants nous aident beaucoup pour que nous nous familiarisions avec l'outil informatique. »

P11 : «Il faut déjà que la personne sache qu'elle va devoir travailler avec les autres via la plateforme. » 


\section{Aspects pédagogiques des $\mathrm{FOAD}$}

Après avoir évoqué les dynamiques en jeu lors du commencement des FOAD, nous pouvons présenter les aspects pédagogiques qui semblent les caractériser. Nous avons notamment identifié l'exigence des FOAD, la collaboration qu'elles semblent développer entre les apprenants, le rôle du tuteur ainsi que la fluctuation de la motivation des apprenants.

\section{Des formations exigeantes}

$\mathrm{Au}$ niveau pédagogique, les FOAD semblent être marquées par des exigences élevées en termes de rythme et de volume de travail :

P5 : « Il faut beaucoup travailler, beaucoup travailler. »

P7 : «C'est vraiment un rythme soutenu. »

$\mathrm{F} 3$ : «C'est des formations très intenses qui demandaient beaucoup, qui étaient très prenantes. »

$\mathrm{F} 1$ : « Ça a été très dur, très dur. »

L'exigence que requièrent les FOAD est alors cumulée à d'autres exigences, telles que l'activité professionnelle et la famille, qui ne sont pas faciles à concilier :

P5 : «Tu as le service, tu as ton foyer, tu as le social, bon... c'est là que ça pose problème. »

P8 : «Les cours, l'emploi du temps du travail, ça n'a pas été facile parce que [...] quand on rentre, on est épuisé. Il faut donc faire un effort pour pouvoir entrer dans les bouquins. »

Cette difficile conciliation se pose d'autant plus lorsque les FOAD incluent des modalités pédagogiques synchrones qui obligent les apprenants à se libérer à un temps donné :

F10 : «Ce n'était pas évident d'être là, dans les «chats » vocaux où les profs étaient en présentiel. »

P7 : «C'est des cours qu'il ne faut pas rater, c'est des rendez-vous où il y a des cours vraiment synchrones que c'est important de suivre. »

Il est possible de penser que les contraintes qu'imposent les modalités pédagogiques synchrones aux apprenants sont très proches des contraintes temporelles des formations en présentiel. Paradoxalement, les apprenants se retrouvent donc confrontés à des contraintes temporelles qu'ils souhaitaient précisément éviter en s'inscrivant à une FOAD plutôt qu'à une formation en présentiel. Il est ainsi possible de penser que les modalités pédagogiques synchrones, bien qu'elles soient sans doute bénéfiques par certains aspects, n'en privent pas moins les apprenants du plus gros avantage des FOAD : leur flexibilité temporelle. 
La difficile conciliation de la famille, de l'emploi et du suivi d'une FOAD implique des sacrifices, notamment sociaux et familiaux :

P1 : «J'ai fait des sacrifices pour mes enfants. Quand je dois rester chez moi pendant le week-end, je reste pour travailler et ce sont les enfants qui sont privés. »

P5 : «Le côté social est aboli parce que tu n'as plus le temps. Tu es tellement occupé, obnubilé par les évaluations. »

P11 : «J'ai dû forcément diminuer mes activités extra-professionnelles, réduire des sorties entre amis, mon temps de télévision. J'ai dû réduire pas mal de choses. »

F9 : «Après l'université, je prenais la fin de toute l'après-midi et la nuit pour travailler. Il y a beaucoup de choses que j'ai dû abandonner. »

Pour répondre aux exigences des FOAD, les participants mettent de l'avant plusieurs traits de caractère essentiels, tels que :

- l'assiduité :

F4: «Je lui [l'apprenant qui commence une FOAD] conseillerais de s'y consacrer au maximum et d'aborder la formation à distance avec le même sérieux qu'une formation en classe. »

F7 : «Il faudrait qu'il [l'apprenant qui commence une FOAD] s'y mette et qu'il ait un emploi du temps assez régulier. »

- l'organisation, notamment la gestion du temps :

P1 : «L'organisation, c'est indispensable. »

P3 : «Pour quelqu'un qui va commencer une formation à distance, je vais lui demander d'être vraiment très bien organisé. »

F9 : «Il suffisait seulement d'être organisé et de se programmer soi-même. »

F11: «Essayer de ne pas [...] perdre son temps. Ça veut dire, organiser son temps, ne pas accumuler. »

- la motivation et la persévérance :

P1 : «Si on est motivé pour faire ceci, on peut y arriver. Si on n'est pas motivé, je ne conseille à aucune personne de se lancer dans une telle formation. »

P6 : «Le premier conseil, c'est d'être courageux parce que ça demande vraiment du courage. Avoir la volonté. »

F10 : «Il faut être super motivé pour suivre et avoir la volonté. »

\section{Des formations à dominante collaborative}

La majorité des FOAD représentées semble beaucoup miser sur les dynamiques de groupe. On trouve en premier lieu l'utilisation fréquente de la modalité pédagogique de travail collaboratif, laquelle est généralement bien reçue par les apprenants même si elle leur demande parfois un temps de familiarisation (cf. partie Un processus de familiarisation) : 
P1 : «Le travail en collaboration, je trouve tout d'abord que ça me permet de gagner beaucoup de temps. [...] Et puis cet échange et cette discussion, ça permet d'avoir un surplus. »

P11: «Les travaux d'équipe, je les aimais bien. C'était des opportunités de partage, de travail collaboratif. »

P2 « Nous n'avons pas assez de compétences et le travail en équipe nous a donné un échange au niveau des compétences, au niveau des atouts, au niveau linguistique, au niveau relationnel même. »

P5: «Je trouve que le groupe de travail a été vraiment bénéfique parce que souvent, tu lis le cours, tu penses avoir compris et la compréhension de l'autre pendant les discussions, ça approfondit ta compréhension. »

P6: «Grosso modo, c'est vraiment une des meilleures ressources de la formation, le travail collaboratif. »

Le travail collaboratif a donc des vertus pédagogiques, mais également motivationnelles :

P3 : «La communication est très importante pour avancer parce que si on est seul à prendre un cours en ligne derrière l'ordinateur, ça peut être ennuyant. »

P7 : «Le fait de travailler en groupe, ça m'a stimulé un peu parce que parfois je me dis que si j'étais seul, il y a des séances que je n'allais pas faire. »

Aussi, nous pouvons poser l'hypothèse que le travail collaboratif représente une alternative appréciée à la dispersion géographique des apprenants qui caractérise les FOAD par rapport au présentiel. L'aspect collaboratif des FOAD semble d'ailleurs dépasser le cadre formel des cours et s'appliquer à des pratiques d'entraide plus informelles initiées par les apprenants :

P12 : «Sur le forum, on se donnait des informations en ce qui concerne le travail, les documentations, on se renvoyait les messages. »

P8 : «Quand ce n'est pas compris, puisque nous sommes une classe virtuelle, on pouvait communiquer entre nous, donc on peut poser la question à un ami qui est dans un autre pays, qui peut nous expliquer. »

F10 : «On s'entraidait. On avait une petite association d'étudiants AEI et c'était vachement sympa. »

F6: «Lorsque nous avions un problème, nous pouvions nous soutenir et demander de l'aide aux autres. »

F8: «On partageait nos recherches, on partageait nos connaissances, on s'envoyait des courriels pour partager ce qu'on avait trouvé ou des questions qu'on avait personnellement sur des exercices. »

Il est intéressant de noter que l'aspect collaboratif informel des FOAD est parfois prolongé en présentiel. Autrement dit, les apprenants à distance d'une même région géographique tendent à doubler la collaboration à distance par des rencontres plus ou moins régulières en présentiel : 
P9 : «On était quatre à Madagascar donc on se voyait tous les quatre. On était six plutôt, mais deux n'ont pas rejoint le groupe donc on était quatre à travailler ensemble. »

F7 : «J'ai rencontré tous les camarades. Nous nous voyions dans la vie. Nous travaillions ensemble au campus. \

F8 : «On était quatre en Côte d'Ivoire et on se voyait souvent sur le Campus numérique ou ailleurs. »

P5 : «On était trois au Burkina donc on a formé un groupe de travail et il y en avait une qui était à l'Université donc on se retrouvait là-bas. On préparait les cours ensemble. »

Le travail collaboratif semble donc pouvoir prendre plusieurs formes, qu'il soit formel ou informel, en distantiel ou en présentiel. Il représente un aspect essentiel des FOAD. Précisons pour finir que cette modalité pédagogique connaît aussi des limites, qu'elles soient dues à des traits de personnalité qui favorisent le travail individuel, à des disponibilités peu conciliables lors de travail en équipe ou à des problèmes relationnels entre les apprenants :

P11 : «Moi, particulièrement j'appréciais mieux le travail individuel parce que je m'organisais mieux, je savais à peu près ce que j'avais à faire et je tenais compte des délais. »

P2 : «Les problèmes d'horaire, parce que parfois, on a une équipe qui se forme de cinq personnes ou bien de quatre personnes ou bien de trois personnes: un Brésilien, une Australienne, un Tunisien et un Français. »

P1 : «On peut se confronter à quelques problèmes entre les coéquipiers. »

P11 : «On avait des étudiants qui ne participaient carrément pas. Ils n'assistaient pas aux réunions synchrones, aux réunions asynchrones, ils ne répondaient pas aux mails... À un moment, on avait l'impression qu'ils nous handicapaient. »

Une part des problèmes relationnels peut d'ailleurs être attribuée à l'aspect multiculturel des groupes d'apprenants inscrits aux FOAD. Autrement dit, certains problèmes relationnels semblent davantage référer à des stéréotypes culturels négatifs ou des interprétations culturelles différentes :

F1 : «Des gens, peut-être ils se disent : "C'est un Algérien, ce n'est pas la peine de discuter avec lui". »

Les problèmes inhérents au travail collaboratif semblent toutefois marginaux et ne suffisent pas à remettre en cause la prédominance positive de cette modalité pédagogique aux yeux des apprenants. Il convient alors de se demander comment se définit le rôle des tuteurs vis-à-vis de la forte collaboration observée entre les apprenants des FOAD. 


\section{Rôle des tuteurs}

Le tuteur semble constituer un aspect pédagogique central des FOAD. Son rôle consiste principalement à :

- délivrer aux apprenants les supports pédagogiques nécessaires à la réalisation des activités et des travaux et indiquer les modalités pédagogiques prévues :

F8 : «On nous déposait le cours sur la plateforme une semaine avant la période de "chat" avec le professeur. C'était pour échanger et partager sur le cours. En même temps, il nous proposait l'exercice à faire et puis il nous divisait en équipe. »

- assurer un suivi, notamment en répondant aux questions des apprenants :

F3 : « Avec nos enseignants, c'était des questions sur un forum. Alors on posait des questions, ils répondaient dans les $48 \mathrm{~h}$ qui suivaient. »

P3 : «J'envoyais vraiment les messages [au tuteur] quand, par exemple, dans notre exercice de groupe à deux, quand on a un problème spécifique. »

Globalement, le rôle des tuteurs s'est avéré positif pour les apprenants, particulièrement lorsqu'ils intervenaient de manière rapide, personnalisée et compréhensive :

P1 : «Personnellement, l'année passée, j'étais très soutenue par deux ou bien trois de mes tuteurs qui étaient sensibilisés aux problèmes que j'ai rencontrés au cours de ma formation. Donc j'ai trouvé la tâche excellente de la part des tuteurs. \

P11 : «On avait des tuteurs très dynamiques qui nous suivaient vraiment. On avait des séminaires, on avait des réunions synchrones. Ils nous relançaient pour des exercices et tout. »

P9 : «Les tuteurs pendant la formation, ils répondent de façon très personnelle à nos attentes, à nos questions. »

F10: «Je leur [les tuteurs] ai envoyé une lettre comme quoi le jeudi et le vendredi, c'était comme samedi et dimanche chez eux et que je ne pouvais pas envoyer. Le prof a été compréhensif. »

F2: «À chaque fois que vous envoyez un "mail", vous êtes sûr d'avoir la réponse du prof à votre prochaine consultation. »

Bien sûr, ce rôle positif des tuteurs n'est possible que si ces derniers sont disponibles. À ce titre, l'indisponibilité des tuteurs est clairement identifiée comme le plus grand reproche rapporté par les participants :

P10 : «Si je prends mon cas simplement, mon tuteur n'a pas été là pour moi. »

P11: «Dès que le cours était mis en ligne, le tuteur disparaissait carrément jusqu'à l'évaluation. »

P12: «Ils ont des cours en présentiel, il y en a qui voyagent beaucoup donc ce n'est pas tout le temps qu'ils ouvrent rapidement leur boîte, qu'ils vont sur la plateforme. »

F4 : «La majorité le faisait [répondre aux questions] de façon tardive. » 
Aussi, nous pouvons conclure que le rôle du tuteur est globalement perçu de façon positive dès lors qu'il se rend disponible pour accompagner les apprenants. Ce faisant, il est en mesure d'intervenir de manière rapide, personnalisée et compréhensive.

\section{Fluctuation de la motivation}

Un autre aspect pédagogique récurrent des FOAD concerne l'évolution de la motivation des apprenants. À cet égard, les exigences des FOAD mentionnées plus haut (cf. partie Des formations exigeantes) sont susceptibles d'altérer la motivation des apprenants :

P2 : «J'avais un certain découragement au départ, dans certains modules de la programmation. »

P5 : « Souvent quand même, quand les mauvaises notes commencent à pleuvoir, à un certain moment, tu te demandes pourquoi tu es venu ici. »

F3: «Après quatre mois, c'était une période de découragement. On avait beaucoup d'informations, on avait beaucoup de travaux. »

Pour remédier à ces baisses de motivation, le tuteur et les pairs semblent jouer un rôle-clé :

P2 : «La motivation de la part du tuteur, l'encouragement et le travail en équipe, ça m'a aidé à résoudre les problèmes de découragement ».

$\mathrm{P} 1$ : «Si je ne paraissais pas dans la plateforme, certains tuteurs me contactaient et me demandaient le pourquoi de cette absence. Si j'avais des problèmes, ils allaient m'aider donc honnêtement, ils m'ont beaucoup soutenue et là je pense qu'ils ont joué une grande part. Si je ne me suis pas découragée, c'est en partie grâce à l'encouragement de mes tuteurs. »

P5 : «Une fois, il y a une collègue qui disait qu'elle allait raccrocher parce qu'elle ne pouvait pas évoluer avec ça. J'ai dit "Non, tu as commencé, tu ne vas pas arrêter, ce n'est qu'un début, peut-être que tu as trébuché, mais tu n'es pas tombée." Donc, on se donnait des conseils et puis on a continué. »

On entrevoit ici, au-delà des échanges pédagogiques et collaboratifs, toute l'importance d'instaurer une relation de qualité entre les apprenants et avec les tuteurs, afin de privilégier une constance motivationnelle.

\section{Aspects technologiques, administratifs et socioprofessionnels des FOAD}

Nous pouvons maintenant aborder d'autres aspects des FOAD, notamment sous l'angle des difficultés qui se posent pour leur bon déroulement. 


\section{Aspects technologiques des FOAD}

Les problèmes liés aux aspects technologiques concernent principalement les pannes du réseau Internet, l'accès difficile à l'équipement informatique et la sousperformance des systèmes informatiques :

P12 : «Un problème, c'est le problème de connexion à Internet. »

F10 : «Jusqu'à présent, il n’y a pas de campus numérique à Djibouti [...]. Suivre la formation, c'est bien, mais encore faut-il avoir les moyens physiques pour continuer cette formation. »

P11 : «J'avais du mal à accéder à la plateforme à partir des cybercafés parce que les machines ne supportaient pas la plateforme. »

Les problèmes d'ordre technologique semblent relativement homogènes chez les participants, contrairement aux problèmes de nature administrative et socioprofessionnelle qui ne concernent que certains pays.

\section{Aspects administratifs des FOAD}

Outre des problèmes administratifs anecdotiques, que nous n'évoquerons pas ici, le problème administratif le plus récurrent chez les participants concerne la nonreconnaissance, par certains pays, des diplômes délivrés par les FOAD. Ce problème n'est pas bénin dans la mesure où il limite les chances d'augmenter l'employabilité des apprenants, ce qui constitue pourtant une de leur motivation à suivre une FOAD (cf. partie Motivation initiale à suivre une FOAD) :

F11 : «C'est juste la reconnaissance, c'est ça le problème qu'on a eu. C'est vrai, j'ai eu mon diplôme, j'ai renouvelé mes compétences. Mais on l'a accroché au mur. Ce n'est pas reconnu.

P2 : «L'accréditation pose un grand problème devant les étudiants parce que quand un étudiant marocain prend son diplôme et voudrait intégrer soit des fonctions publiques soit des formations, le diplôme n'est pas accrédité. »

Dans la même lignée, la non-reconnaissance des diplômes issus des FOAD diminue la pertinence et, dans son prolongement, la viabilité des FOAD dans les pays concernés :

$\mathrm{P} 2$ : «Si le Maroc arrive à bien régler ce problème de statut de la validation des diplômes, le e-learning va connaître un essor. »

F1 : «Tant qu'il n'y a pas de reconnaissance des diplômes de la formation à distance, tant qu'on n'est pas reconnu, ça ne marche pas. »

Au vu de ce problème, il semble essentiel de travailler avec les pays concernés à la reconnaissance des acquis développés via les FOAD. 


\section{Aspects socioprofessionnels des FOAD}

«Du fait de la non reconnaissance administrative des diplômes des FOAD, ces dernières apparaissent alors marginales sur le plan socioprofessionnel :

P10 : «Ce n'est pas quelque chose qui est vraiment vulgarisé. Ça ne court pas les rues. »

P2 : «Même au niveau de l'université, les gens sont très peu informés. Même les professeurs d'université les plus titrés ne savent en quelque sorte même pas de quoi il s'agit quand on leur parle d'une formation à distance. »

P8 : «Les institutions ne connaissent pas. Ce n'est pas trop vulgarisé en Côte d'Ivoire. Les gens ne connaissent pas la formation à distance en tant que telle. »

Étant marginales, les FOAD souffrent éventuellement de préjugés socioprofessionnels négatifs :

P3 : «Ils ignorent, c'est pourquoi ils ont peut-être un mauvais préjugé. Les gens ne savent pas suffisamment et ils ne savent pas non plus comment ça se déroule. »

F4: «L'école ne se mobilise pas dans le sens de montrer que la formation à distance est aussi efficace que la formation en présentiel. Ils disent: "Ah, la formation à distance, ce n'est pas important". »

F7 : «Ce [les FOAD] n'est pas bien perçu. »

À l'instar du manque de reconnaissance au niveau administratif, les apprenants confrontés à ce problème se voient privés de l'augmentation de l'employabilité qui les motive en partie à suivre une FOAD dans la mesure où leur diplôme est sousvalorisé. À cet égard, pour que les FOAD actualisent leur plein potentiel, il semble important de sensibiliser le marché du travail des pays concernés aux qualifications réelles qu'elles permettent.

\section{Retombées des FOAD}

Les retombées des FOAD sont abordées par les participants principalement en termes de bénéfices professionnels. Les deux principaux d'entre eux correspondent point pour point aux motivations des apprenants à s'inscrire à une FOAD (cf. partie Motivation initiale à suivre une FOAD), ce qui semble indiquer que les FOAD répondent adéquatement aux attentes des apprenants. On retrouve donc :

- des bénéfices sur le plan du développement de compétences professionnelles :

P5 : «En tous cas, ça m'a permis de développer des compétences. »

P6 : «Je me suis beaucoup, beaucoup amélioré par rapport aux années passées et j'en suis très fier. »

P8 : « Ça m’a permis de développer des compétences. » 
- une augmentation de l'employabilité :

F2 : «Des profs m'impliquent dans plein de choses parce que simplement ils sentent que j'ai d'autres compétences, alors que ces compétences, je les ai acquises à travers ces formations-là. »

F6 : «J'ai des compétences, ce qui me rend plus compétitif sur le plan professionnel.

F9 : «Sur le plan professionnel, ça m'a donné une bonne position. »

L'augmentation de l'employabilité se traduit pour certains par un avancement professionnel concret :

P4 : « J'ai même déjà des demandes, des offres d'emploi qu'on me fait. »

P7 : «Ça m’a permis de créer mon entreprise. »

F6 : « Je n'ai pas eu de problèmes à me trouver un emploi après la formation. »

F12 : «J'ai pu avoir de l'avancement professionnel. »

Un autre bénéfice tiré des FOAD, bien que moins rapporté, consiste à contribuer au développement des ressources humaines qui peuvent ensuite être mises au service des pays africains :

F2 : «Je me suis rendu compte que ça doit être un processus irréversible qui permet aux pays africains d'améliorer la qualité de leurs ressources humaines sans qu'ils ne soient exposés à la fuite des cerveaux. »

F4 : «Cela pourra apporter une contribution au développement de l'Afrique, dans le domaine éducatif. »

F9 : «C'est pour dire que cette formation m'a été très très utile, non seulement pour mon doctorat, mais aussi parce que partout où je passe, je parle d'environnement et je suis dans une ONG pour la défense de l'environnement et dans une autre pour la défense des Droits de l'Homme où l'on a pris en compte le volet "environnement". »

Au vu des bénéfices qu'ils en ont tirés, l'ensemble des participants fait état d'une appréciation hautement positive des FOAD :

P10 : «Dans l'ensemble, j’ai apprécié. Ça a été une expérience gratifiante pour ma part.

P12 : «Ça en vaut la peine. »

$\mathrm{P} 2$ : «Si je dois faire un bilan global, il est positif. »

P5 : «En tant que professionnel, je tire beaucoup d'avantages de la formation à distance. »

P6 : « Le bilan, il est très riche. »

P7 : «J'ai vraiment trop aimé la formation à distance que j'aurais de la difficulté à déceler des défauts.

F10 : « Sincèrement, j’ai beaucoup apprécié la formation à distance. » 
F3 : « Je ne regrette pas de l'avoir choisi de toutes les façons. »

F4 : «Les formations à distance, c'est passionnant. »

F7 : « Je suis très fière des formations que $\mathrm{j}$ 'ai suivies et je ne regrette rien. Je suis très contente de ces formations par rapport à ce que ça m'apporte. »

\section{Discussion et conclusion : où va donc la distance ?}

Nous pouvons maintenant revenir sur les résultats obtenus en lien avec la thématique de ce numéro : où va la distance ? À cet égard, plusieurs des distances évoquées dans le cadre conceptuel de cet article semblent faire écho à cette étude. La distance spatiale, par exemple, lorsque les participants aux FOAD n'ont pas un Centre numérique de l'AUF à proximité, ni l'équipement nécessaire à la maison. La distance technologique également, qui se retrouve dans le dysfonctionnement ou la vétusté de l'équipement et de l'infrastructure technologique dont disposent les apprenants pour suivre leur FOAD. Ce type de distance se fait également sentir dans la familiarisation technologique que demande initialement le suivi d'une FOAD. De la même manière, la distance pédagogique apparaît pour plusieurs apprenants non familiers avec certaines formules pédagogiques employées dans les FOAD, notamment la collaboration. À ce titre, il est possible de penser qu'une introduction approfondie au fonctionnement technique des plateformes des FOAD et aux formules pédagogiques utilisées permettrait de réduire d'emblée ces deux types de distance. La distance temporelle se manifeste, quant à elle, de manière inattendue. En effet, dans les résultats obtenus, c'est davantage les temps de formation en synchrone qui semblent potentiellement problématiques dans la mesure où ils imposent aux participants une contrainte temporelle difficilement conciliable avec la vie familiale et professionnelle des apprenants. En un sens, c'est donc davantage la recherche d'une proximité temporelle qui semble poser problème (bien qu'elle revête sans doute des avantages par ailleurs), et non la distance temporelle en tant que telle. Enfin, la distance socioculturelle est relativement discrète dans les résultats obtenus. Elle semble apparaître dans certains conflits d'origine interculturelle entre les apprenants, mais ceux-ci sont apparemment peu courants. On la retrouve également dans la non-reconnaissance, par certains pays, des diplômes obtenus par les FOAD, lesquelles souffrent éventuellement de certains préjugés défavorables à leur endroit. Pour réduire ce type de distance, il apparaitrait nécessaire de travailler avec les pays concernés à la reconnaissance des diplômes issus des FOAD et de sensibiliser le marché du travail de ces mêmes pays aux qualifications réelles que les FOAD permettent. Globalement, il est toutefois possible de penser que les différentes distances évoquées ci-dessus ne semblent pas porter atteinte au bon déroulement des FOAD, ni à la satisfaction des apprenants à leur égard.

Pour répondre à la question « Où va la distance ? », nous pouvons donc avancer, au vu de nos résultats, qu'elle va résolument vers de nouveaux espaces (l'Afrique dans notre cas), et ce, dans un mouvement d'internationalisation qui forme une 
tendance actuelle majeure des institutions universitaires. Ceci pose, en retour, la question de la dimension interculturelle des FOAD, qui semble encore timidement prise en compte dans la conception et dans l'animation des FOAD, et qui méritera sans doute d'être approfondie par la suite pour les optimiser.

\section{Bibliographie}

Akkari A., «Au-delà de l'ethnocentrisme en sciences de l'éducation», P. R. Dasen et C. Perregaux (dir.), Pourquoi des approches interculturelles en sciences de l'éducation?, Bruxelles, De Boeck Université, 2002.

Albirini A., "The Internet in developing countries: A medium of economic, cultural and political domination", International Journal of Education and Development using ICT, vol. $4, \mathrm{n}^{\circ}$ 1, 2008. http://ijedict.dec.uwi.edu/viewarticle.php?id=360\&layout=html

Allen B. A., et Boykin A. W., "African-American Children and the Educational Process: Alleviating Cultural Discontinuity Through Prescriptive Pedagogy", School Pschology Review, vol. 21, n 4, 1992, p. 586-596.

Blanchard E., et Frasson C., «Amélioration de la motivation par support à l'autonomie et prise en compte des cultures dans les systèmes de e-Learning », Pierre S. (dir.), Innovations et tendances en technologies de formation et d'apprentissage. Montréal, Presses internationales de Polytechnique, 2005.

Brossard M., et Foko B., Coûts et financement de l'enseignement supérieur en Afrique francophone, Série Développement humain de la région Afrique, Banque mondiale, 2007. http://siteresources.worldbank.org/INTAFRREGTOPEDUCATION/Resources/444659-1 210786813450/Enseignement_superieur_cout_financement_fr.pdf

Cassady J-C., Mohammed A., et Mathieu L., "Cross-Cultural Differences in Test Perceptions: Women in Kuwait and the United-States", Journal of Cross-Cultural Psychology, vol. 35, $n^{\circ} 6,2004$, p. 713.

Cervantes M., et Guellec D., "The brain drain: old myths, new realities", OECD Observer, $\mathrm{n}^{\circ}$ 230, 2002. http://www.oecdobserver.org/news/fullstory.php/aid/673/The_brain_drain: _Old_myths,_new_realities.html

Docquier F., «Fuite des cerveaux et inégalités entre pays», Revue d'économie $d u$ développement, vol. $2, \mathrm{n}^{\circ}$ 21, 2007, p. 49-88.

Gilbert W. A., Retention in distance education telecourses and perceptions of faculty contact: A comparison of traditional and nontraditional community college students. Thèse de doctorat inédite, Florida State University, Talahassee, Floride, 2000.

Gioan P-A., Enseignement Supérieur en Afrique Francophone. Quels leviers pour des politiques financièrement soutenables?, Document de travail de la Banque mondiale $\mathrm{n}^{\circ}$ 103, 2007. http://siteresources.worldbank.org/EDUCATION/Resources/278200-1099 079877269/547664-1099079956815/AFTHD_WPS103.pdf

Harry K., Higher Education Through Open and Distance Learning: World review of distance education and open learning, London, Routledge, 1999. 
Henderson L., "Instructional Design of Interactive Multimédia: A Cultural Critique", Educational Technology Research and Development, vol. 44, n 4, 1996, p. 85-104. DOI: 10.1007/BF02299823

Huberman A. M., Miles M. B., Analyse des données qualitatives. Recueil de nouvelles méthodes, Brussels, Belgique, De Boeck, 1991.

Institut statistique de l'UNESCO, Recueil de données mondiales sur l'éducation 2009 : statistiques comparées sur l'éducation dans le monde, 2009. http://www.uis.unesco.org/ Library/Documents/ged09-fr.pdf

Intsiful J., Okyere P. F., et Osae S., "Use of ICT for education, research and development in Ghana : Challenges, opportunities and potentials", Communication présentée au 2003 Round Table on Developing Countries Access to Scientific Knowledge, The Abdus Salam ICTP, Trieste, Italie, 2003.

Jacquinot G., «Apprivoiser la distance et supprimer l'absence ? ou les défis de la formation à distance », Revue française de pédagogie, $\mathrm{n}^{\circ} 102$, 1993, p. 55-67.

Karsenti T., et Collin S., «Quelle place pour les TIC en formation initiale d'enseignants de français? Le cas de l'Afrique », Revue internationale des technologies en pédagogie universitaire, vol. $7, \mathrm{n}^{\circ} 3,2010$, p. 32-47.

L'Écuyer R., Méthodologie de l'analyse développementale du contenu. Méthode GPS et concept de soi, Québec, QC, Presses de l’Université du Québec, 1990.

Meyer J-B., et Charum J., «La "fuite des cerveaux" est-elle épuisée ? Paradigme perdu et nouvelles perspectives », Cahiers des sciences humaines, vol. 31, n 4, 1995, p. 1003 1019.

Mott V. W., "Is Adult Education an Agent for Change or an Instrument of the Status Quo?", S.B. Merriam, B. C. Courtenay et R. M. Cervero (dir.), Global Issues and Adult Education: Perspectives from Latin America, Southern Africa and the United-States, San Francisco, Jossey-Bass, 2006.

Moughli L., Semporé J., Koné T. G., «Formation en maintenance et gestion des infrastructures et équipements communaux en Afrique. D'une formation en présence à une formation à distance », Distances et savoirs, vol. 6, $\mathrm{n}^{\circ}$ 2, 2008, p. 237-249.

OCDE, La cyberformation dans l'enseignement supérieur: synthèse, 2006. http://www. oecd.org/dataoecd/54/49/35961190.pdf

Oladele B. A., "The imperatives of challenges for Africa in the knowledge age: Status and role of national information policy", Communication présentée au $67^{\text {th }}$ IFLA Council and General Conference, Boston, États-Unis, 2001.

Polikanov D., et Abramova I., “Africa and ICT: A chance for breakthrough?”, Information, Communication and Society, vol. 6, $\mathrm{n}^{\circ}$ 1, 2003, p. 42-56.

Selinger M., "The Imfundo Project: ICT in teacher education in developing countries", J. Price, D. A. Willis, N. Davis et J. Willis (dir.), Proceedings of Society for Information Technology \& Teacher Education International Conference (SITE), 2001, p. 3008-3013. Chesapeake, VA: Association for the Advancement of Computing in Education (AACE).

Sfez L., Technique et idéologie, un enjeu de pouvoir, Paris, France, Seuil, 2002. 
514 D\&S - 9/2011. Où va la distance ?

Tiemtoré W. Z., «Les TIC dans l'éducation en Afrique subsaharienne: espoir fondé de développement ou émergence d'une nouvelle utopie ", Cahier de recherche du centre d'intérêt scientifique M@rsouin, 6, 2007. http://recherche.telecom-bretagne.eu/labo_ communicant/projet/

Tunca B., Barriers in using technology. Dans P. Barker et S. Rebelsky (dir.), Proceedings of World Conference on Educational Multimedia, Hypermedia and Telecommunications, 2002, p. 1980-1982. Chesapeake, VA : Association for the Advancement of Computing in Education (AACE).

UNESCO, Note d'information sur « L'enseignement à distance » abordé au cours de la $38^{\mathrm{e}}$ semaine (29/05-04/06/2006) de célébration du 60e anniversaire de l'UNESCO, 2006. http://www.unesco.org/bpi/pdf/memobpi38_distancelearning_fr.pdf

Union africaine, Seconde décennie de l'éducation pour l'Afrique, Plan d'action (2006-2015). http://www.adea-comed.org/IMG/pdf/deuxieme_decennie_education_pour_afrique_plan _daction.pdf 